Revista Destaques Acadêmicos, Lajeado, v. 11, n. 3, 2019. ISSN 2176-3070

DOI: http://dx.doi.org/10.22410/issn.2176-3070.v11i3a2019.2293

http://www.univates.br/revistas

\title{
AVALIAÇÃO NUTRICIONAL E PERFIL SÓCIO DEMOGRÁFICO DE IDOSOS INSTITUCIONALIZADOS
}

\author{
Elisabete Bald ${ }^{1}$, Fernanda Scherer Adami ${ }^{2}$
}

Resumo: Objetivo: O presente estudo objetivou relacionar o perfil sócio demográfico e o estado nutricional de idosos institucionalizados de um município do Vale do Taquari, Rio Grande do Sul. Metodologia: O estudo foi do tipo transversal, com amostra composta por 58 idosos residentes há mais de um ano em duas Instituições de Longa Permanência e, para a obtenção das variáveis sociodemográficas foi utilizado um questionário estruturado com perguntas fechadas. Para avaliar o estado nutricional do idoso foi calculado o IMC e classificado conforme OPAS (Organização Panamericana de Saúde, 2002). Para análise estatística foi utilizado o Teste de associação Exato de Fisher, calculado pelo software SPSS versão 22.0 e os resultados foram considerados significativos a um nível de significância máximo de 5\%. Resultados: A maioria da amostra era do sexo feminino 65,5\%, com idade entre 80 e 90 anos 48,3\% e 43,3\% eram eutróficos. a via de alimentação oral e ensino médio completo foram significativamente associados à instituição sem fins lucrativos e o uso da sonda e ensino fundamental completo à instituição com fins lucrativos, $(p=0,048)$ e $(p=0,026)$, respectivamente. Conclusão: A maioria dos idosos, eram mulheres, tinham mais de 80 anos e eram eutróficos. Os idosos com alimentação oral e ensino médio completo foram significativamente associados à instituição sem fins lucrativos e o uso da sonda e ensino fundamental completo à instituição com fins lucrativos.

Palavras-chave: Idosos, envelhecimento, qualidade de vida, estado nutricional.

\section{INTRODUÇÃO}

De acordo com a Organização Mundial da Saúde (OMS), o aumento da longevidade da população é uma das maiores conquistas da humanidade e um dos maiores desafios mundiais (OMS, 2016). O processo de envelhecer traz em si muitas incertezas, principalmente no que se refere às condições de saúde, e os cuidados com a pessoa idosa, com isso muitas famílias tendem a dividirem as

1 Nutricionista, elisabete.bald@hotmail.com

2 Nutricionista, Doutora em Ambiente e Desenvolvimento e docente na Universidade do Vale do Taquari - Univates. fernandascherer@univates.br 
responsabilidades do cuidado desses indivíduos com as Instituições de Longa Permanência para Idosos (ILPI), pois o envelhecimento acarreta a diminuição da capacidade funcional, a alterações neurológicas, nutricionais e anatômicas e juntamente com o sedentarismo pode resultar em prejuízos á saúde e à qualidade de vida dos idosos, levando a perda de autonomia, baixa autoestima e depressão (SILVÉRIO et al., 2016).

O ritmo de crescimento acelerado do número de idosos pode ser acompanhado pelas mudanças severas nas estruturas e nos padrões familiares, o que reflete em menos pessoas disponíveis para cuidar dessa população idosa que vem crescendo a cada dia (OMS 2005). Esse processo de envelhecer traz em si muitas incertezas, principalmente no que se refere às condições de saúde, e os cuidados com a pessoa idosa. Com isso muitas famílias tendem a dividirem as responsabilidades do cuidado desses indivíduos com as ILPI (SOUZA et al., 2014).

A verificação do estado nutricional é primordial para se identificar indivíduos em risco de desnutrição, sendo estes os mais suscetíveis a doenças. A avaliação nutricional realizada por métodos antropométricos é provavelmente a mais utilizada devido às suas vantagens como custo acessível, simplicidade e de fácil aquisição, dessa forma, podem-se auxiliar os profissionais no tratamento para a recuperação e promoção da saúde dos idosos (SPEROTTO; SPINELLI, 2010).

Portanto, sabemos que a institucionalização, assim como as inúmeras mudanças decorrentes do envelhecimento, afeta significativamente a capacidade de tomada de decisões dos idosos, além disso, podem reduzir a qualidade de vida ou dificultar a manutenção do peso saudável (RAMOS et, al, 2012).

Diante disto, o presente estudo objetivou relacionar o perfil sócio demográfico e o estado nutricional de idosos institucionalizados de um município do Vale do Taquari, Rio Grande do Sul (RS).

\section{METODOLOGIA}

O estudo foi do tipo transversal, descritivo e analítico, com 51 idosos institucionalizados há mais de um ano, em duas instituições de Longa Permanência para idosos de um município do Vale do Taquari, RS.

Para a obtenção das variáveis sociodemográficas foi utilizado um questionário estruturado com perguntas fechadas sobre sexo, idade, categorizada em 60 aa 70 anos, de 70 a 80 anos e acima de 80 anos, escolaridade, classificada como analfabeto, ensino fundamental, ensino médio, ensino superior, estado civil classificado como solteiro ou separado, casado e viúvo, e religião categorizada como católica, evangélica, outra, e sem religião, tipo de instituição classificada como com fins lucrativos e sem fins lucrativos, tipo de 
alimentação (oral ou sonda) e consistência da alimentação (normal, pastosa ou líquida por sonda naso entérica).

A avaliação do estado nutricional foi realizada por meio do Índice de Massa Corporal (IMC) e classificado conforme a recomendação da Organização Pan-americana da Saúde (OPAS, 2002). Para a medida do peso, o idoso utilizou vestimentas padronizadas, ficando descalço e posicionando-se em pé na balança digital da marca Filizola ${ }^{\circledR}$, com capacidade para $150 \mathrm{Kg}$. Para a medida da estatura, o participante da pesquisa, descalço, foi posicionado com os pés lado a lado, encostando a parte posterior do corpo em uma fita métrica inelástica, fixada na parede sem rodapé, sendo estes os pontos de corte adotados para idosos no Brasil segundo recomendações do Sistema de Vigilância Alimentar e Nutricional (SISVAN, 2011).Nos idosos acamados, as medidas de peso e estatura serão obtidas por meio das equações de estimativas (CHUMLEA et al., 1987).

Caso a pesagem não seja possível, o peso corporal é estimado por meio de fórmulas de estimativa de peso para idosos que utilizam a altura do joelho (AJ), dobra cutânea subescapular (DCSE), circunferência do braço (CB), circunferência da panturrilha $(\mathrm{CP})$ e circunferência abdominal (CA) (SOUZA et al., 2013).

O estudo atendeu à resolução $n^{\circ} 466 / 2012$, sendo aprovado pelo COEP da Universidade do Vale do Taquari - Univates sob número 91028618.4.0000.5310.

Os dados obtidos através dos questionários foram tabulados em uma planilha do Excel e para análise estatística foi utilizado o Teste de associação Exato de Fisher. Os resultados foram considerados significativos a um nível de significância máximo de $5 \%(\mathrm{p} \leq 0,05)$ e o software utilizado para esta análise foi o SPSS versão 22.0 .

\section{RESULTADOS}

A idade média dos idosos foi de 78,4 $\pm 9,1$ anos, média da CP de 34,7 $\pm 6,8 \mathrm{~cm}$ e do IMC de $28,0 \pm 8,2 \mathrm{Kg} / \mathrm{m}^{2}$.

Na Tabela 1 verificou-se que a maioria 51,7\% (30) residia em Instituições sem fins lucrativos, $65,5 \%$ (38) eram mulheres, $48,3 \%$ (28) tinham mais de 80 anos, $62,1 \%$ (36) eram viúvos e $67,2 \%$ (39) relataram ensino fundamental completo. Quanto a via e consistência da alimentação, 93,1\% (54) alimentam-se por via oral, consistência normal $75,9 \%$ (44) e apresentam estado nutricional de eutrofia $43,1 \%$ (25).

Tabela 1. Caracterização do tipo de instituição, sexo, idade, estado nutricional, via de alimentação, consistência da alimentação, estado civil, escolaridade, e religião

\begin{tabular}{llll}
\hline Variável & Resposta & $\mathrm{N}^{\circ}$ casos & $\%$ \\
\hline
\end{tabular}




\begin{tabular}{|c|c|c|c|}
\hline \multirow[t]{2}{*}{ Tipo de Instituição } & Com fins lucrativos & 28 & 48,3 \\
\hline & Sem fins lucrativos & 30 & 51,7 \\
\hline \multirow[t]{2}{*}{ Sexo } & Feminino & 38 & 65,5 \\
\hline & Masculino & 20 & 34,5 \\
\hline \multirow[t]{3}{*}{ Idade (anos) } & 60 a 70 anos & 12 & 20,7 \\
\hline & $70-80$ anos & 18 & 31,0 \\
\hline & Mais de 80 anos & 28 & 48,3 \\
\hline \multirow[t]{4}{*}{ Estado Nutricional } & Baixo Peso & 10 & 17,2 \\
\hline & Eutrófico & 25 & 43,1 \\
\hline & Sobrepeso & 8 & 13,8 \\
\hline & Obesidade & 15 & 25,9 \\
\hline \multirow[t]{2}{*}{ Via de alimentação } & Oral & 54 & 93,1 \\
\hline & Sonda & 4 & 6,9 \\
\hline \multirow[t]{3}{*}{$\begin{array}{l}\text { Consistência da alimen- } \\
\text { tação }\end{array}$} & Normal & 44 & 75,9 \\
\hline & SNE & 5 & 8,6 \\
\hline & Pastosa & 9 & 15,5 \\
\hline \multirow[t]{3}{*}{ Estado Civil } & Solteiro/Separado & 17 & 29,3 \\
\hline & Casado & 5 & 8,6 \\
\hline & Viúvo & 36 & 62,1 \\
\hline \multirow[t]{2}{*}{ Escolaridade } & Ensino Fundamental & 39 & 67,2 \\
\hline & Ensino Médio & 19 & 32,8 \\
\hline \multirow[t]{3}{*}{ Religião } & Católica & 45 & 77,6 \\
\hline & Evangélica & 11 & 19,0 \\
\hline & Não tem & 2 & 3,4 \\
\hline
\end{tabular}

Na Tabela 2 evidenciou-se que a via de alimentação oral e ensino médio completo foram significativamente associados à instituição sem fins lucrativos e o uso da sonda e ensino fundamental completo à instituição com fins lucrativos, $(p=0,048)$ e $(p=0,026)$, respectivamente.

Tabela 2. Associação do sexo, faixa etária, estado nutricional, via de alimentação, consistência da alimentação, estado civil, escolaridade e religião com o tipo de Instituição de Longa Permanência

\begin{tabular}{|c|c|c|c|c|c|c|}
\hline \multirow[b]{3}{*}{ Variável } & \multirow[b]{3}{*}{ Resposta } & \multicolumn{4}{|c|}{ Instituição } & \multirow[b]{3}{*}{$\mathrm{P}$} \\
\hline & & \multicolumn{2}{|c|}{$\begin{array}{l}\text { Com fins } \\
\text { lucrativos }\end{array}$} & \multicolumn{2}{|c|}{$\begin{array}{l}\text { Sem fins } \\
\text { lucrativos }\end{array}$} & \\
\hline & & $\mathrm{N}$ & $\%$ & $\mathrm{~N}$ & $\%$ & \\
\hline Sexo & Feminino & 17 & 60,7 & 21 & 70,0 & 0,582 \\
\hline
\end{tabular}




\begin{tabular}{|c|c|c|c|c|c|c|}
\hline & Masculino & 11 & 39,3 & 9 & 30,0 & \\
\hline \multirow[t]{3}{*}{ Idade (anos) } & 60 a 70 & 7 & 25,0 & 5 & 16,7 & 0,061 \\
\hline & $70-80$ & 12 & 42,9 & 6 & 20,0 & \\
\hline & Mais de 80 & 9 & 32,1 & 19 & 63,3 & \\
\hline \multirow{4}{*}{$\begin{array}{l}\text { Classificação } \\
\mathrm{IMC} \mathrm{Kg} / \mathrm{m}^{2}\end{array}$} & Baixo Peso & 6 & 21,4 & 4 & 13,3 & 0,811 \\
\hline & Eutrófico & 12 & 42,9 & 13 & 43,3 & \\
\hline & Sobrepeso & 4 & 14,3 & 4 & 13,3 & \\
\hline & Obesidade & 6 & 21,4 & 9 & 30,0 & \\
\hline \multirow{2}{*}{$\begin{array}{l}\text { Via de } \\
\text { alimentação }\end{array}$} & Oral & 24 & 85,7 & 30 & 100,0 & 0,048 \\
\hline & Sonda & 4 & 14,3 & - & - & \\
\hline \multirow[t]{3}{*}{ Consistência } & Normal & 21 & 75,0 & 23 & 76,7 & 0,289 \\
\hline & SNE & 4 & 14,3 & 1 & 3,3 & \\
\hline & Pastosa & 3 & 10,7 & 6 & 20,0 & \\
\hline \multirow[t]{3}{*}{ Estado Civil } & Solteiro/Separado & 9 & 32,1 & 8 & 26,7 & 0,093 \\
\hline & Casado & - & - & 5 & 16,7 & \\
\hline & Viúvo & 19 & 67,9 & 17 & 56,7 & \\
\hline \multirow[t]{2}{*}{ Escolaridade } & Ensino Fundamental & 23 & 82,1 & 16 & 53,3 & 0,026 \\
\hline & Ensino Médio & 5 & 17,9 & 14 & 46,7 & \\
\hline \multirow[t]{3}{*}{ Religião } & Católica & 23 & 82,1 & 22 & 73,3 & 0,749 \\
\hline & Evangélica & 4 & 14,3 & 7 & 23,3 & \\
\hline & Não tem & 1 & 3,6 & 1 & 3,3 & \\
\hline
\end{tabular}

Teste Exato de Fisher

Não foi observada associação significativa entre faixa etária, via de alimentação, consistência da alimentação, estado civil, escolaridade e religião com o estado nutricional dos idosos institucionalizados (TABELA 3).

Tabela 3. Associação do sexo, faixa etária, via de alimentação, consistência da alimentação, estado civil, escolaridade e religião com o estado nutricional dos idosos das Instituições de Longa Permanência

\begin{tabular}{|c|c|c|c|c|c|c|c|c|c|c|}
\hline \multirow[b]{3}{*}{ Variável } & \multirow[b]{3}{*}{ Resposta } & \multicolumn{8}{|c|}{ Classificação IMC } & \multirow[b]{3}{*}{$\mathrm{P}$} \\
\hline & & \multicolumn{2}{|c|}{ Baixo Peso } & \multicolumn{2}{|c|}{ Eutrofia } & \multicolumn{2}{|c|}{ Sobrepeso } & \multicolumn{2}{|c|}{ Obesidade } & \\
\hline & & $\mathrm{N}$ & $\%$ & $\mathrm{~N}$ & $\%$ & $\mathrm{n}$ & $\%$ & $\mathrm{n}$ & $\%$ & \\
\hline \multirow[t]{2}{*}{ Sexo } & Feminino & 6 & 60,0 & 15 & 60,0 & 4 & 50,0 & 13 & 86,7 & 0,204 \\
\hline & Masculino & 4 & 40,0 & 10 & 40,0 & 4 & 50,0 & 2 & 13,3 & \\
\hline \multirow[t]{3}{*}{ Idade (anos) } & 60 a 70 anos & 2 & 20,0 & 3 & 12,0 & 4 & 50,0 & 3 & 20,0 & 0,069 \\
\hline & $70-80$ anos & 6 & 60,0 & 8 & 32,0 & - & - & 4 & 26,7 & \\
\hline & $\begin{array}{l}\text { Mais de } 80 \\
\text { anos }\end{array}$ & 2 & 20,0 & 14 & 56,0 & 4 & 50,0 & 8 & 53,3 & \\
\hline
\end{tabular}




\begin{tabular}{|c|c|c|c|c|c|c|c|c|c|c|}
\hline \multirow{2}{*}{$\begin{array}{l}\text { Via de } \\
\text { alimentação }\end{array}$} & Oral & 8 & 80,0 & 24 & 96,0 & 7 & 87,5 & 15 & 100,0 & \multirow[t]{2}{*}{0,164} \\
\hline & Sonda & 2 & 20,0 & 1 & 4,0 & 1 & 12,5 & - & - & \\
\hline \multirow[t]{3}{*}{ Consistência } & Normal & 4 & 40,0 & 19 & 76,0 & 7 & 87,5 & 14 & 93,3 & \multirow[t]{3}{*}{0,058} \\
\hline & SNE & 2 & 20,0 & 2 & 8,0 & 1 & 12,5 & - & - & \\
\hline & Pastosa & 4 & 40,0 & 4 & 16,0 & - & - & 1 & 6,7 & \\
\hline \multirow[t]{3}{*}{ Estado Civil } & $\begin{array}{l}\text { Solteiro/ } \\
\text { Separado }\end{array}$ & 5 & 50,0 & 5 & 20,0 & 3 & 37,5 & 4 & 26,7 & \multirow[t]{3}{*}{0,614} \\
\hline & Casado & 0 & 0,0 & 3 & 12,0 & 1 & 12,5 & 1 & 6,7 & \\
\hline & Viúvo & 5 & 50,0 & 17 & 68,0 & 4 & 50,0 & 10 & 66,7 & \\
\hline \multirow[t]{2}{*}{ Escolaridade } & Ensino Fund. & 7 & 70,0 & 13 & 52,0 & 6 & 75,0 & 13 & 86,7 & \multirow[t]{2}{*}{0,144} \\
\hline & Ensino Médio & 3 & 30,0 & 12 & 48,0 & 2 & 25,0 & 2 & 13,3 & \\
\hline \multirow[t]{3}{*}{ Religião } & Católica & 9 & 90,0 & 16 & 64,0 & 7 & 87,5 & 13 & 86,7 & \multirow[t]{3}{*}{0,224} \\
\hline & Evangélica & - & - & 8 & 32,0 & 1 & 12,5 & 2 & 13,3 & \\
\hline & Não tem & 1 & 10,0 & 1 & 4,0 & - & - & - & - & \\
\hline
\end{tabular}

Teste Exato de Fisher

\section{DISCUSSÃO}

O processo de envelhecimento ocorre de forma particular para cada individuo e pode influenciar no estado nutricional do idoso, ocasionar a mortalidade precoce ou agravar os problemas de saúde (CORTEZ; MARTINS, 2012).

As mulheres institucionalizadas foram maioria neste estudo, resultado semelhante a outros estudos com idosos institucionalizados por Azevedo et al. (2007) que descreveram o perfil de 80 octogenários de uma cidade do interior de São Paulo, onde observou-se que 72,5\% eram mulheres idosas e 27,5\% eram homens idosos. Menezes e Maruci (2005), também constataram um predomínio na população idosa feminina.

O número de mulheres idosas é maior que o de homens em todos os grupos etários e essa diferença torna-se ainda maior com o aumento da idade (75 anos e mais), em consequência das maiores taxas de mortalidade experimentadas pelos homens e à maior longevidade das mulheres (ALVES et al., 2004). Esse fato refere-se a menor exposição das mulheres a riscos que determinam a mortalidade por causas externas, a procura mais sistemática por serviços de saúde ao longo de toda a vida e ainda por menor consumo de bebidas alcoólicas e tabaco quando comparadas aos homens (PONTES et al., 2009). Esse fato refere-se a menor exposição das mulheres a riscos que determinam a mortalidade por causas externas, a procura mais sistemática por serviços de saúde ao longo de toda a vida e ainda por menor consumo de bebidas alcoólicas e tabaco quando comparadas aos homens (PONTES et al., 2009). 
Em relação ao perfil dos idosos, evidenciou-se que a maioria apresentava idade entre 80 e 89 anos, mostrando similaridade ao estudo realizado por Rauen et al. (2008), onde predominaram os idosos com mais de 80 anos. O aumento do número de idosos com mais de 80 anos é um importante fator na institucionalização, devido à maior tendência no comprometimento da condição de saúde desses, especialmente no aspecto da dependência (RAUEN et al., 2008). Pois a institucionalização está, na maioria das vezes, associada a uma maior dependência física e cognitiva (TALMELLI et al., 2010). Nesses casos, a família alega falta de recursos financeiros, espaço físico insuficiente ou pouca disponibilidade de tempo para o cuidado ao idoso, sendo encaminhado então o mesmo para uma instituição, mesmo contra sua vontade (MARIN et al., 2012).Outra característica apontada no presente estudo se refere à predominância de idosos viúvos, resultado semelhante ao estudo realizado em Rio Grande, no RS, com 153 residentes em ILPI, (BRANDÃO et al., 2008). Tanto em instituição sem fins lucrativos como em instituição com fins lucrativos na cidade de Porto Alegre, observou-se que a carência familiar, viuvez e solidão foram os principais motivos de asilamento para ambos os locais de estudo (TERRA et al., 2009).

No atual estudo, 43,1\% apresentou eutrofia, porém $17,2 \%$ foi diagnosticado com desnutrição, resultado diferentes ao encontrado por Ramos et al. (2012), que realizou uma avaliação nutricional com pacientes idosos em atendimento domiciliar, onde foi observado que $49 \%$ dos pacientes eutróficos, $48 \%$ com risco de desnutrição e apenas 3\% com desnutrição. Ramos et al. (2012) também observou que idosos institucionalizados apresentam uma maior prevalência de desnutrição em comparação aos idosos que vivem em suas moradias fixas, semelhantes aos valores encontrados por Paula et al. (2007) que verificaram por meio do IMC, uma prevalência de desnutrição em $36,6 \%$ dos 260 idosos de um hospital em SC. Com isso percebe-se que a população do presente estudo, por ter percentuais inferiores de desnutrição está mais protegida em relação ao estado nutricional, já que a manutenção do estado nutricional adequado pode interferir positivamente, permitindo uma maior expectativa e uma melhor qualidade de vida desta população (SANTOS et al., 2014).

A via de alimentação oral foi significativamente associada a instituição sem fins lucrativos e o uso da sonda à instituição com fins lucrativos. De acordo com Volpini, Frangella (2013), as alterações na consistência da dieta podem estar relacionadas às modificações estruturais, morfológicas e bioquímicas próprias do envelhecimento, como a mudança na composição muscular, redução da salivação e problemas dentários, e uso de próteses dentárias mal adaptadas. Entretanto, salienta-se que a modificação na consistência da dieta em indivíduos muito idosos, geralmente, faz-se necessária também pela dificuldade de deglutição e / ou pelo risco de aspiração de alimentos (VOLPINI; FRANGELLA, 2013). 
O ensino médio completo foi associado à instituição sem fins lucrativos ensino fundamental à instituição com fins lucrativos. a elevação da escolaridade, e consequentemente do nível sócio econômico proporciona um maior acesso aos bens e serviços e interferem na possibilidade e/ou ao acesso aos alimentos, afetando nas escolhas alimentares ao longo da vida (MARUCCI; BARBOSA, 2003).

Não foi observada associação significativa entre faixa etária, no atual estudo com o estado nutricional, resultado diferente do estudo realizado por Luís et al. (2011), que concluiu que a idade dos participantes em seu estudo foi inversamente proporcional à classificação do estado nutricional, ou seja, quanto maior a idade há um declínio no estado nutricional.

A limitação do estudo está relacionada ao reduzido número amostral podendo não refletir a situação regional dos idosos institucionalizados, mas pode ser utilizado como indicador para o planejamento de ações de promoção à saúde entre os idosos das instituições estudadas.

\section{CONCLUSÃO}

Concluiu-se com este estudo que a maioria dos idosos residia em Instituições sem fins lucrativos, eram mulheres, tinham mais de 80 anos, viúvos, relataram ensino fundamental completo, eutróficos e quanto a via e consistência da alimentação alimentam-se por via oral. Os idosos com alimentação oral e ensino médio completo foram significativamente associados à instituição sem fins lucrativos e o uso da sonda e ensino fundamental completo à instituição com fins lucrativos.

Com base nestes resultados encontrados no presente estudo, fica evidenciado a importância da realização de uma avaliação nutricional rotineira por parte da equipe de saúde da ILPI, visto que os idosos institucionalizados representam uma população vulnerável aos distúrbios nutricionais, tanto pelas condições impostas pela institucionalização, como pelas condições sócio demográficas e fator idade.

\section{REFERÊNCIAS}

AZEVEDO Luciane Coutinho de; FENILLI Manoela; FARIAS, MAURA Bagnolin; BREITKOPF, Thayse; SILVA, Amanda Alcaraz da; ESMERALDINO, Roberto.

Principais fatores da mini-avaliação nutricional associada a alterações nutricionais de idosos hospitalizados. Arquivos Catarinenses de Medicina, v. 36, n. 3, Santa Catarina, 2007. Disponível em: < http:/ /bases.bireme.br/cgi-bin/wxislind.exe/iah/ online/>. Acesso em: 25 de maio de 2019.

BARRETO, João. Envelhecimento e qualidade de vida: o desafio atual. Revista de Sociologia, v.15, p. 289-302, cidade do Porto, 2005. Disponível em: < http:/ /ojs.letras. 
up.pt/index.php/Sociologia/article/view/2393/2190>. Acesso em: 10 de julho de 2019.

BRANDÃO Aline Ferreira. Estado nutricional e características sócio-econômicodemográficas de idosos institucionalizados na cidade do Rio Grande, RS. Dissertação (Mestrado em Enfermagem). Programa de Pós-Graduação em Enfermagem, Universidade Federal do Rio Grande, Rio Grande do Sul, 2008. Disponível em: < http:/ / repositorio.furg.br/bitstream/handle/1/2948/alinebrandao. pdf?sequence=1>. Acesso em: 10 de julho de 2019 .

CHUMLEA, Cameron et al. Prediction of body weight for the nonambulatory elderly from anthropometry. Journal of the American Dietetic Association, v. 88, n. 5, p. 564-8. 1987. Disponível em: < https:/ / www.ncbi.nlm.nih.gov/pubmed/3367012>. Acesso em: 10 de julho de 2019.

CORTEZ Antônio Carlos Leal, MARTINS Maria do Carmo de Carvalho. Indicadores antropométricos do estado nutricional em idosos: uma revisão sistemática. Revista UNOPAR Cient Ciênc Biol Saúde, v.14, nº 4, p.271, Piauí, 2012. Disponível em: < http:/ / revista.pgsskroton.com.br/index.php/JHealthSci/article/view/887/851>. Acesso em: 02 de julho de 2019.

DE LUIS, D. A. et al. Evaluation of the mini-nutritional assessment short-form (MNA-SF) among institutionalized older patients in Spain. Nutr. Hosp., v. 26, n. 6, p.1350- 1354. Madrid, 2011. Disponível em: <http:/ / scielo.isciii.es/pdf/nh/ v26n6/23_original_10.pdf>. Acesso em: 30 de junho de 2019.

MARIN, Maria José Sanchez et al. Compreendendo a História de Vida de idosos institucionalizados. Rev. Bras. Geriatr. Gerontol, v. 15, n. 1, p.147-154, Rio de Janeiro, 2012. Disponível em:< http:/ / www.scielo.br/pdf/rbgg/v15n1/16.pdf > . Acesso em: 24 junho de 2019.

MARUCCI, Maria de Fátima Nunes; BARBOSA, Aline Rodrigues. Estado nutricional e capacidade física. In: SABE - Saúde, bem-estar e envelhecimento; O projeto SABE no município de São Paulo: uma abordagem inicial [S.l: s.n.], 2003. Disponível em: < https:/ /bdpi.usp.br/single.php?_id=002307119>. Acesso em: 30 de junho de 2019.

MENEZES, T. N. de; MARUCCI, M. de F. N. Oferta e consumo alimentar de idosos residentes em instituições geriátricas: diferença no valor energético total. Revista Nutrire SBAN - Sociedade Brasileira de Alimentação e Nutrição, v. 31, n. 2, p. 1-11, Fortaleza/Ceará, 2006. Disponível em: <http:/ / sban.cloudpainel.com.br/files / revistas_publicacoes/126.pdf>. Acesso em: 30 de junho de 2019.

OMS. ORGANIZAÇÃO MUNDIAL DA SAÚDE. [Capturado em 14 de julho de 2019]. Disponível em: <http://www.who.int/countries/bra/es/>. Acesso em 14 de julho de 2019.

ORGANIZAÇÃO PAN-AMERICANA DE SAÚDE (OPAS). XXXVI Reunión del Comitê Asesor de Investigaciones en Salud - Encuestra Multicêntrica - Salud Beinestar y Envejecimeiento (SABE) en América Latina e el Caribe - Informe 
preliminar. Disponível em: <http:/ / www.imsersomayores.csic.es/documentos / documentos/paho-salud-01.pdf >. Acesso em: 30 de junho de 2019

PAUlA H. A. A., OLIVEIRA F. C. E., SÃO JOSÉ J. F. B, GOMIDE C.L., ALFENAS R.C.G. Avaliação do estado nutricional de pacientes geriátricos. Rev. Bras. Nutr. Clin., v.22, n.4, p. 280-5, Viçosa/MG, 2007. Disponível em: <https:/ / www. researchgate.net/publication/242749015_Avaliacao_do_estado_nutricional_de_ pacientes_geriatricos>. Acesso em: 20 de julho de 2019.

PONTES, R.J.S. et al. Transição Demográfica e Epidemiológica In: MEDRONHO, R.A. et al. Epidemiologia. 2.ed. São Paulo: Atheneu, 2009. p. 123- 151. Disponível em: < file:/ / D:/Downloads/322-Texto\%20do\%20artigo-1490-1-10-20170127.pdf>. Acesso em: 10 de julho de 2019.

RAMOS, Luciana Junqueira et al. Aspectos éticos e nutricionais em uma amostra de idosos institucionalizados e não-institucionalizados. Revista do Hospital de Clínicas de Porto Alegre, v. 32, n. 2, Porto Alegre, 2012. Disponível em: < https:/ / seer.ufrgs. br/hcpa/article/view/30740>. Acesso em: 25 de junho de 2019.

RAUEN, Michelle. Soares et al. Avaliação do estado nutricional de idosos institucionalizados. Rev. Nutr., v. 21, n.3, p.303-310, Campinas, 2008. Disponível em: <http:/ / www.scielo.br/scielo.php?script=sci arttext\&pid=S1415-52732008000300005> . Acesso em: 25 de junho de 2019.

SANTOS, K. T et al. Indicadores Antropométricos de Estado Nutricional como Preditores de Capacidade em Idosos. Revista Brasileira de Medicina do Esporte, v. 20, n. 3, São Paulo, SP, 2014. Disponível em: < http:/ / www.scielo.br/pdf/rbme/ v20n3/1517-8692-rbme-20-03-00181.pdf>. Acesso em: 02 de julho de 2019.

SILVÉRIO, Jane Kátia A.; PEDREIRA, Katherine Rios A.; KUTZ, Nyvian Alexandre; SALGUEIRO, Marcia Maria Hernandes A. Estado nutricional de idosos institucionalizados: uma revisão de literatura. Revista: Visão Acadêmica; v. 17, n. 3, p. 75-90, 2016. Disponível em: <file:/ / / D:/Downloads/49477-193460-2-PB.pdf>. Acesso em: 20 de maio de 2019

SOUZA, Rafaela; FRAGA, Juliana S.; BERTASSO, Catarina; GOTTSCHALL, Andreatta; BUSNELLO, Fernanda M.; RABITO, Estela I. Avaliação antropométrica em idosos: estimativas de peso e altura e concordância entre classificações de IMC. Rev. Bras. Geriatr. Gerontologia. V. 16; N. 1; Pág. 81-90, Rio de Janeiro, 2014. Disponível em: < http:/ /www.scielo.br/pdf/rbgg/v16n1/a09v16n1.pdf>. Acesso em: 30 de maio de 2019.

SPEROTTO F.M.; SPINELLI R. B. Avaliação nutricional em idosos independentes de uma instituição de longa permanência no Município de Erechim - RS. Revista Perspectiva, v. 4, n. 125, p. 105-116, Erechim, 2010. Disponível em: < http:/ / www. uricer.edu.br/site/pdfs/perspectiva/125_78.pdf >. Acesso em: 10 de junho de 2019.

TALMELLI, L. F. et al. Nível de independência funcional e déficit cognitivo em idoso com doença de Alzheimer. Rev. Esc. Enferm. USP, v. 44, n. 4, p. 933-9, São 
Paulo, 2010. Disponível em:< http:/ / www.scielo.br/pdf/reeusp/v44n4/11.pdf>. Acesso em: 24 de junho de 2019.

TERRA, N. L. et al. Diferenças biopsicossociais entre idosos de instituição asilar particular e filantrópica da cidade de Porto Alegre. Scientia Medica, v. 19, n. 1, p. 3-10, Porto Alegre, 2009. Disponível em: < file:/ / / D:/Downloads /4188-42373-1PB\%20(1).pdf>. Acesso em: 13 de julho de 2019.

VOLPINI, Milena Maffei; FRANGELLA, Vera Silvia. Avaliação nutricional de idosos institucionalizados, Centro Universitário São Camilo - São Paulo, SP, Brasil,

Ainstein (São Paulo), v.11, n.1, São Paulo, 2013. Disponível em: <http:/ / www.scielo. br/pdf/eins/v11n1/a07v11n1.pdf>. Acesso em: 20 de junho de 2019. 